\section{MRS and SMM held International Materials Research Congress 2012 in Cancún}

www.mrs-mexico.org.mx/imrc2012

www.mrs.org/meeting-scene

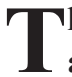

he XXI International Materials Research Congress (IMRC) 2012, chaired by Orlando Auciello of Argonne National Laboratory, USA; J. Gerardo Cabañas Moreno of Instituto Politécnico Nacional, Mexico; Sandra Rodil Posada of Instituto de Investigaciones en Materiales (UNAM), Mexico; and Francesco Stellacci of École Polytechnique Fédérale de Lausanne, Switzerland, was held in Cancún, Mexico,

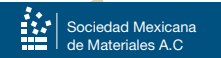
on August 12-17. IMRC 2012 was a joint effort of the Materials Research Society (MRS)

MRS and the Sociedad Mexicana de Materiales (SMM). In-depth coverage of the plenary sessions, technical talks, and other meeting events is available at www.mrs.org/meeting-scene.

For materials researchers who decide to enter the world of the entrepreneur, plenary speaker Carlos A. Paz de Araújo (Symetrix Corp.; University of Colorado, USA) said, "You must try everything to keep your original spirit-your love for science." Araújo provided a blueprint for scientists to become technological entrepreneurs. He succeeded in this environment by turning things upside down, he said, starting with "search and development," a process in which a researcher must find an important problem that is possible to solve. This is then followed by research, development, and marketing. Following this technique, Araújo developed ferroelectric random-access memory devices that are now used in cars, cell phones, televisions, radiofrequency tags, and printers. Even after achieving success, researchers must reinvent themselves periodically to keep their interest in science alive, Araújo said, as he proceeded to explore his next research project, which also addressed memory devices.

Plenary speaker Cecilia Noguez of the Universidad Nacional Autónoma de México in Mexico City discussed the light interaction with metal nanoparticles that have extinction, scattering, and absorption components, all of which differ in relative magnitude depending on the nature of the material, as well as particle sizes and shapes. Surface plasmon resonances are responsible for absorption of light. All of these variables enable the manipulation of light at the nanoscale for many different applications. In medicine, for example, plasmonic phenomena are involved in the photothermal destruction of cancer cells. In energy, improved photovoltaic devices can be obtained from exploiting plasmonic properties.

The work of Bao-Lian Su's teams at the Wuhan University of Technology, China, and at the University of Namur, Belgium, on living hybrid materials also finds applications in the energy and health fields. Following his mantra of "do as nature, work as nature, and produce as nature," $\mathrm{Su}$ and his colleagues are working on systems that directly use natural systems in combination with artificial materials. For example, they used silica-based porous systems with chloroplasts, thyalkoides, plant cells from $A$. thaliana, and cyanobacteria to produce artificial leaves for clean energy photosynthesis. In the health field, they are trying to synthesize living hybrid materials to treat diabetes and other diseases.

At Rutgers University in New Jersey, Manish Chhowalla and his co-workers are producing two-dimensional sheets of transition-metal dichalcogenides and graphene oxide (GO) using solution
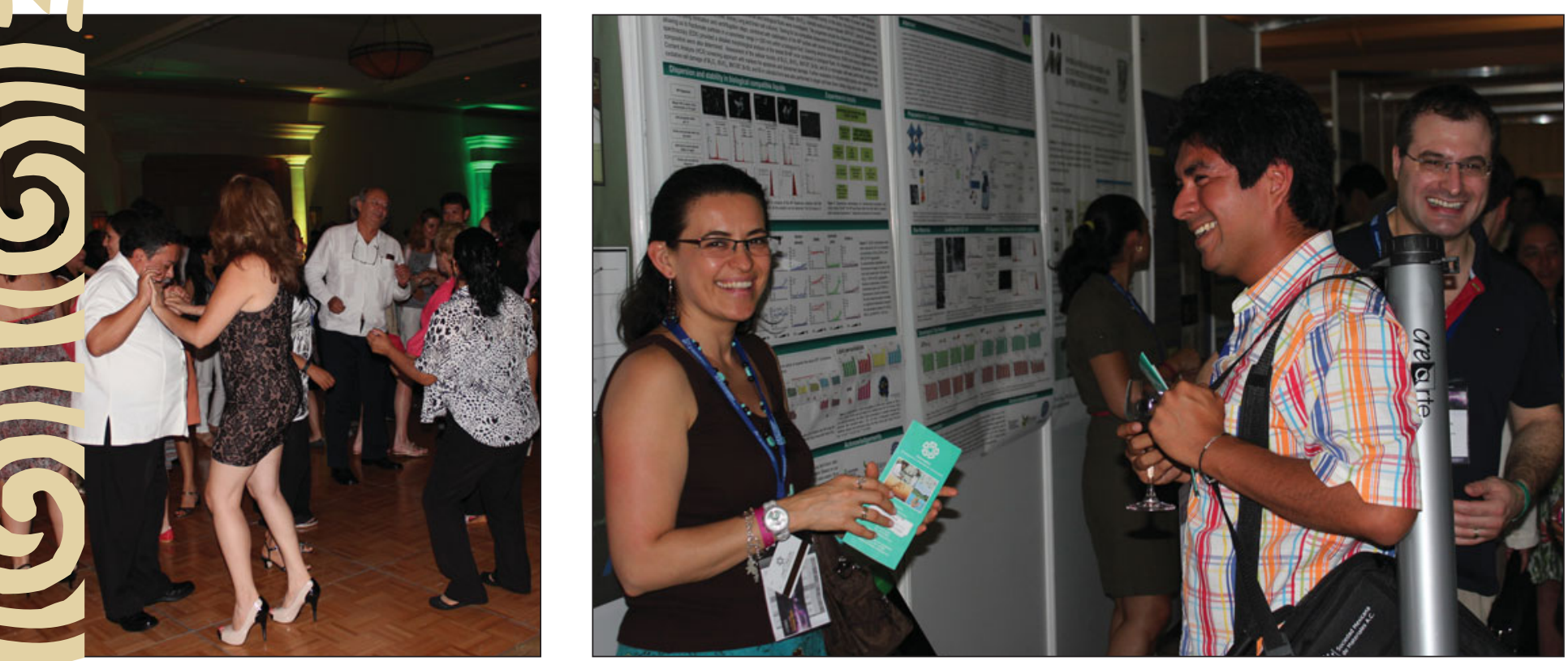
processable methods, which could lead to large-scale production of these materials for applications in electronics, catalysis, and energy storage. In his plenary address, Chhowalla demonstrated GO films as electrodes in the solution-processable light-emitting diodes and as a hole-transport layer in organic photovoltaics. He also demonstrated that singlelayered transition-metal dichalcogenides are excellent catalysts for hydrogen evolution and potentially useful for optoelectronics.

Monica Olvera de la Cruz of Northwestern University, in her plenary address, connected Platonic and Archimedean geometries to materials science. These geometries occur spontaneously in crystalline shells having more than one component. When the two components have different elastic moduli, the shell buckles in ways that depend on the relative concentrations of the components. From the sheer number of different shapes that de la Cruz showed, it appears that the variety of buckling geometries is endless. "Our goal is to explore the consequences of this faceting of ionic membranes and find other shapes," she said. This experimental and computational work is leading to a deeper understanding of the geometries of various cellular microcompartments including viruses, organelles, and wall envelopes of halophilic organisms, as well as many crystalline materials.

For a change of pace, Luis Rodríguez, Professor Emeritus of the Center for Radio Astronomy and Astrophysics at the National University of Mexico, spoke at the elegant Science Luncheon where he showed the benefits of studying astronomy through the means of radio wavelengths. His images revealed that galaxies viewed through radio wavelengths appear to be much larger than when seen in the visible range. Persuaded to study the universe outside of the optical wavelengths, from radio waves to gamma rays, astronomers and astrophysicists have discovered pulsars, gamma-ray bursts, and the cosmic microwave background, among other interesting phenomena.

Of the over 1200 technical presenta- tions given by researchers representing 40 countries, some addressed the areas of energy efficiency, organic materials for electronics and photonics, and biomaterials and biomedical devices. Sarbajit Banerjee and his group at the University of Buffalo are using the phase change of vanadium oxide $\left(\mathrm{VO}_{2}\right)$ to develop thermochromic window coatings for smart windows. $\mathrm{VO}_{2}$ undergoes a phase change from a monoclinic crystal structure to a tetragonal one at $67^{\circ} \mathrm{C}$ over only $300 \mathrm{fs}$. This change is technologically important because the monoclinic phase is transparent to infrared (IR) radiation, while the tetragonal is IR-reflective. The smart windows always let in visible sunlight. However, at low temperatures they also let in the IR, which warms up a room, while at higher temperatures they block IR, keeping the room cool.

Polymers can also be effective in optoelectronics applications. Specifically, design and synthesis of nonlinear organic chromophores for photonics and optoelectronics is of much interest for their possible applications in photorefractive polymers. In the work presented by J. Apolinar Muñoz-Rodríguez (Centro de Investigaciones en Óptica, León, Mexico), a new Schiff base with a D- $\pi$-A architecture was synthesized. Following synthesis and characterization, the films were placed between transparent indium tin oxide deposited on glass slides, and an external electric field was applied. Muñoz-Rodríguez showed how fast, reversible holographic imaging was carried out using these polymer films.

In the biological realm, talks ranged from the nano- to the macroscale. Andrea de Vizcaya Ruiz, a toxicologist in Cinvestav, Mexico reported that although nanomaterials are attractive for a number of medical applications, characteristics such as increased residence time, access to the central nervous system, and their increased surface-to-volume ratio are drawbacks that could lead to their application becoming more harmful than healing. For these reasons, de Vizcaya Ruiz said, there is an increasing need to establish the potential toxicity and health impacts that nanomaterials could impose, from their design and function-

\author{
Sponsors of the XXI \\ International Materials \\ Research Congress (IMRC) 2012 \\ AKT \\ Asylum Research \\ Blue Wave Semiconductors \\ Bruker \\ CEMEX \\ Centro de Investigación en Química \\ Aplicada-CIQA \\ Centro de Investigaciones en Óptica \\ Consejo Nacional de Ciencia y Tecnología- \\ CONACYT \\ GLOBAL Office of Naval Research, Science \\ and Technology \\ Graphene Laboratories \\ Instituto de Investigaciones en \\ Materiales-IIM \\ Instituto Potosino de Investigación \\ de Cientifica y Tecnología-IPICYT \\ Intercovamex \\ National Science Foundation \\ NT-MDT \\ PANalytical \\ Seki Diamond \\ Shinshu University \\ Universidad de La Ciénega del Estado de \\ Michoacán de Ocampo \\ University of South Florida \\ Wiley-VCH
}

alization in the laboratory to their application and use.

On the biological macroscale, David Stout of Brown University addressed the search for a cardio-patch or "bandaid" for the heart to help repair it after a heart attack. Using carbon nanofibers, his group worked toward creating a biodegradable, polymer-based cardio-patch that is conductive and would promote growth of heart cells (cardiomyocytes). By adding highly purified carbon nanofibers with diameters ranging $100-300 \mathrm{~nm}$ to nonconducting poly(lactic glycolic acid) in various ratios, the researchers formed composite materials that show much promise as a cardio-patch, Stout said.

Further news coverage on the plenary and broad array of technical sessions as well as other special activities can be accessed online through the MRS Meeting Scenes: www.mrs.org/meeting-scene. $\square$ 


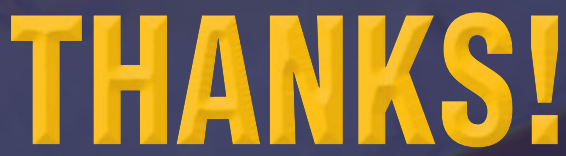

The following events at the $\mathbf{2 0 1 2}$ MRS Fall Meeting have been funded, in part, by the generous contributions of these organizations.

\section{Kurt J. Lesker}

www.lesker.com • Booth 400

pubs.acs.org • Booth 125

\section{COFFEE BREAKS}

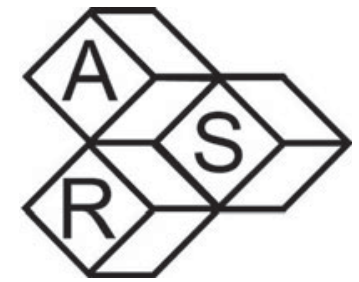

www.arscryo.com • Booth 304

\section{WOMEN IN MATERIALS SCIENCE BREAKFAST}

\section{(4) $A L D$ materials science}

www.sigma-aldrich.com/matsci • Booth 1024

\section{BADGE LANYARDS}

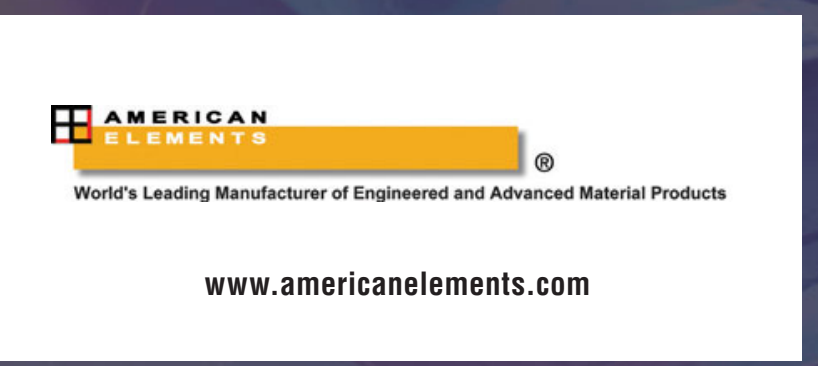


AJA International, Inc.

www.ajaint.com

Sputtering Systems; Sputter Sources; Sputter Targets

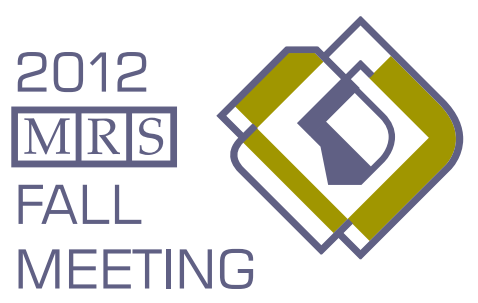

Hynes Convention Center - Level 2

Tuesday, November $27 \cdot$ 11:00 am - 5:30 pm Wednesday, November $28 \cdot 11: 00 \mathrm{am}-6: 00 \mathrm{pm}$ Thursday, November $29 \cdot 10: 00 \mathrm{am}-1: 30 \mathrm{pm}$

10 Angstroms

Booth 323

www.10angstroms.com

Electron Beam Lithography Systems;

Nanoindenters; Refurbished SEMs

\section{A \& N Corporation}

www. ancorp.com

Vacuum Chambers; Vacuum Fittings; Vacuum Valves

\section{Across International LLC}

Booth 802

www.acrossinternational.com

Induction Heaters; Ball Mills; Ovens

\section{ACS Publications}

pubs.acs.org

ACS Nano; Nano Letters; Journal of Physical

Chemistry C; ACS Applied Materials \& Interfaces

\section{AdValue Technology, LLC}

Booth 622

www.advaluetech.com

Alumina Product; Quartzware; Zirconia Products

\section{Advanced Polymer Materials Inc. Booth 1207}

www.apmpolymers.com

Block Copolymers; Functional Copolymers;

Conductive Polymers

\section{Advanced Research Systems, Inc.}

Booth 304

www.arscryo.com

Closed and Open Cycle Cryocooler; Cryogenic Probe

Station; Helium Liquefiers

\section{Agilent Technologies \\ Booth 401 \\ www.agilent.com/find/nano}

Nanoindenters; Universal Testing Systems; Field

Emission Scanning Electron Microscopes; Atomic

Force Microscopes

\section{AIP Publishing}

Booth 117

www.aip.org

Physics Journals; Online Hosting;

Conference Proceedings

\section{AIST-NT, Inc.}

Booth 1308

www.aist-nt.com

Atomic Force/Scanning Probe Microscopes;

Combined AFM \& Raman Spectroscopy Systems

\section{AIXTRON SE}

Booth 507

WWW.aixtron.com

MOCVD/CVD/PECVD Equipment; OVPD and PVPD

Equipment; AVD and ALD Equipment

\section{AldLab Chemicals LLC}

Booth 1305

www.aldlabnano.com

Nanowires; Nanoparticles; Nanotech

Formulation and Drug Delivery

\section{Aldrich Materials Science \\ www.sigma-aldrich.com/matsci \\ Organic Electronics; Nanomaterials;}

Booth 1024

Alternative Energy

\section{Alfa Aesar, a Johnson}

Matthey Company

Booth 912

www.alfa.com

High-Purity Metals; Evaporation Materials; Ceramics

\section{Alfred University}

engineering.alfred.edu

Education; Research

\section{Alicat Scientific, Inc.}

www.alicatscientific.com

Mass Flow Control (MFCs); Pressure \& Differential Pressure Control

\section{Amastan LLC}

www.amastan.com

Nanomaterials; Nanomaterial Manufacturing

Equipment; Spheroidization

American Physical Society

Booth 225

publish.aps.org

Publications; Physics Journals; Online Journals

ANA Innovation Huts and Services Inc. Booth 320 www.anarghyainnotech.com

Confocal Raman AFM; UHV ESCA/XPS/ARPES; UHV

Components and Systems, Cryo UHV AFM/STM

\section{Anasys Instruments Corp.}

Booth 621

www.anasysinstruments.com

AFM+; Nanoscale Infrared Spectroscopy

(nanolR); nano-TA2

Andeen-Hagerling, Inc.

www.andeen-hagerling.com

Precision Capacitance and Loss Bridges; Precision Reference Capacitors

\section{Anfatec Instruments AG}

www.anfatec.de

SPM Controllers; LockIn Amplifiers

AFM/Kelvin Probe

\section{Angstrom Engineering Inc.}

www.angstromengineering.com

CVD, PVD, Sputter Deposition Systems; Thermal

Evaporation Systems; Electron Beam Systems;

Glovebox Integrated Thin Film Technologies

\section{Angstrom Sciences Inc.}

www.angstromsciences.com

Booth 1307

Magnetrons; Sputtering Material;

Sputtering Cathodes

\section{Angstrom Scientific Inc.}

Booth 707

www.angstrom.us

Electron Microscopy; Nano-manipulators

Angstrom Sun Technologies, Inc.

Booth 931

www.angstec.com

Spectroscopic Ellipsometer; Microspectrophotometer Film Thickness Tool

\section{Annealsys}

Booth 806

www.annealsys.com

RTP; RTCVD; Annealing; MOCVD; Spray-CVD; LPCVD
Apple Academic Press, Inc.

www.appleacademicpress.com

Booth 1302

Professional/Academic Books

Applied NanoStructures, Inc.

Booth 1206

wWw.appnano.com

AFM Probes; MEMS; Microscope Parts

Applied Surface Technologies

Booth 609

www.co2clean.com

$\mathrm{CO}_{2}$ Snow Jet Cleaning; Surface Cleaning;

Substrate Cleaning

Arradiance, Inc.

www.arradiance.com

ALD Systems; ALD Services; ALD-Activated

Microchannel Plates and Detectors

Asahi Spectra Co., Ltd.

www.asahi-spectra com

Xenon Light Source; Solar Simulator; Optical Filter

\section{ASMEC GmbH}

www.asmec.de

Nano Hardness Tester; Nanoindenter; Analysing

Software

\section{Asylum Research}

Booth 510

www.AsylumResearch.com

Atomic Force/Scanning Probe Microscopes:

Nanoindenter

\section{attocube systems $A G$}

Booth 509

www attocube.com

Nanopositioning Systems; Low-vibration

Cryogen-free Cryostats; Low Temperature Scanning

Probe and Confocal Microscopes; Fiber-based Interferometric Sensor Systems

Attolight AG

Booth 720

www attolight.com

Cathololuminescence System; SEM

AVS

www.avs.org

Publishing; Education; Membership

B\&W Tek, Inc.

www.bwtek.com

Raman Spectrometers; Spectrometer Modules; Lasers

Baden-Württemberg International Booth 111

www.bw-i.de

Discover Scientific Excellence Made in Germany and Explore Career Opportunities

Balazs NanoAnalysis,

a Division of Air Liquide

www balazs com

Analytical Testing; Materials Characterization AMC-SMC

BaySpec, Inc.

Booth 832

www.bayspec.com

Raman Microscope; Raman Moving Lab; Raman

Benchtop 1064, 785, 532

BigC: DinoLite Scopes

www.bigc.com

Handheld Digital Microscopes

Biolin Scientific, Inc.

www.biolinscientific.com

Quartz Crystal Microbalance with Dissipation

Monitoring; Farfield Dual Polarization Interferometer;

Attension Theta Optical Tensiometer

Join us for a

Wine \& Cheese

Happy Hour Reception

on Wednesday

from 5:00 to $6: 00 \mathrm{pm}$ 
Bio-Logic USA, LLC

Booth 907

www.bio-logic.us

Electrochemical Research; Potentiostats;

Fuel Cell/Battery Testing

Blue Wave Semiconductors, Inc.

Booth 803

www.bluewavesemi.com

Substrate Wafer Heaters; Thin Film Deposition

Systems; Thin Films and Coating Materials;

R\&D Services

Bluestone Global Tech, Inc.

Booth 1200A

www bluestonegt com

Graphene Film; Graphene on Wafer; Graphene-based

Device and Application

Brooks Automation, Inc.

Booth 722

www.brooks.com

835 Vacuum Quality Monitor System; XC Cryochiller

\section{Bruker}

Booth 408

www.bruker-axs.com

$X$-Ray Diffraction

\section{Bruker Nano Surfaces Division}

Booth 409

www.bruker.com

Atomic Force Microscopes; 3D Optical

Microscopes; Stylus Profilers; Tribometers;

Nano-Micro- and Macro-Indenters; Scratch

Testers; AFM Probes

Cambridge NanoTech Inc.

Booth 205

Www.cambridgenanotech.com

Atomic Layer Deposition; Thin Film Deposition;

Ultra Thin Coatings

Cambridge University Press

Booth 105

www.cambridge.org/us

Books; Journals

Capovani Brothers Inc.

Booth 1314

www capovani.com

Used Scientific Equipment

\section{Carl Zeiss Microscopy, LLC}

www.zeiss.com/microscopy

Light Microscopes; Electron Microscopes;

Cameras \& Software

Chemat Technology, Inc.

Booth 201

www.chemat com

Spin Coating; Dip Coating; Chemical Precursors

ColdEdge Technologies, Inc.

Booth 719

www coldedgetech com

Cryostats; Cryocoolers; Cryogenics

COMSOL, Inc.

Booth 705

wWW.comsol.com

COMSOL Multiphysics

CRAIC Technologies, Inc.

www.microspectra.com

Microspectrophotometers; Raman

\section{Cryogenic Limited}

www.cryogenicusa com

Superconducting Magnets; Measurement Systems;

Cryogen Free Systems

\section{Crystal Bank}

crystalbank.com

Single Crystals; Copper Single Crystals; Substrates

\section{CRYSTAL GmbH}

www.crystal-gmbh.com

Substrate/Wafer; Laser Crystals;

Optical Components

CrystalMaker Software Ltd.

Booth 501

www crystalmaker com

CrystalMaker; CrystalDiffract; SingleCrystal
CSM Instruments Inc.

Booth 617

www.csm-instruments.com

Scratch Testers; Indentation Testers; Pin-on-Disk

Tribometer; Testing Services

cyberTECHNOLOGIES USA, LLC

Booth 729

www.cybertechnologies.com

Surface Quality Measurement Systems;

Flatness \& Thickness Measurement Systems;

Total Thickness Measurement Systems

DCA Instruments, Inc.

Booth 520

www dca fi

MBE Systems; PLD Systems; UHV Sputter Systems

\section{DELMIC BV}

Booth 1125

www.delmic.com

SPARC System; SECOM Platform

\section{Delong America Inc.}

www.lv-em.com

Benchtop Combined TEM and SEM; Low Voltage TEM

Denton Vacuum, LLC

Booth 913

www dentonvacuum com

Sample Preparation Tools; High Vacuum Carbon

Evaporators; Thin Film Deposition Systems

\section{Digital Surf SARL}

Booth 704

www.digitalsurf.com

Imaging and Analysis Software for Light Microscopes,

SPMs, SEMs, Spectrometers

EBARA Technologies, Inc.

Booth 809

www.ebaratech.com

Vacuum Pumps; Turbo Pumps

\section{Ecopia Corp.}

Booth 321

www ecopia21.co.kr

Variable Temperature Hall Effect Measurement

System; Probe Station; RTP System

eDAQ, Inc.

Booth 823

www.edaq.com

High Resolution Impedance Analysis; Tethered

Membrane Analysis; General Data Recording

Electron Microscopy Sciences

Booth 307

www.emsdiasum.com

Laboratory Supplies; Chemicals/Adhesives Equipment

Elsevier

Booth 101

www.elsevier.com

Books; Journals; Electronic Products

EM4SYS Co., Ltd.

Booth 1118

www.em4sys.com

AFM; Nano State; Nano Measurement

Energetiq Technology, Inc.

www.energetiq.com

UV Light Source; Broadband Light Source;

High Brightness Light Source

European Synchrotron Radiation

Booth 219

Facility, Institut Laue Langevin

www.esrf.eu

Synchrotron X-ray and Neutron Facilities

\section{FEl Company}

Booth 311

www.fei.com

Scanning Electron Microscopes; Transmission

Electron Microscopes; DualBeam SEM/FIB

Microscopes

First Nano, a division

Booth 702

of CVD Equipment Corporation

www.firstnano.com

CVD Equipment; Gas/Chemical Delivery Systems;

Gas Abatement Systems; CVD Materials

Fischer Technology, Inc.

www.fischer-technology.com

Fischerscope XRF; Microhardness Testers
Fischione Instruments

Booth 508

www.fischione.com

Electron Microscope Accessories; Sample Preparation

Flow Sciences, Inc.

www flowsciences com

Glove Box; Isolater; Safety Hood

FM Lab

www.fmlab.ru

F-Mobile Energy; Energy Storage; Functional Materials

FUJIFILM Dimatix, Inc.

www.dimatix.com

Dimatix Materials Printer; Dimatix Materials Cartridge Other Dimatix Printheads \& Systems

\section{Gamry Instruments}

www.gamry.com

Potentiostats; Quartz Crystal Microbalance

Electrochemistry Accessories

Gatan, Inc.

Booth 301

www.gatan.com

Materials Characterization; Nanotechnology

Photovoltaics

Geib Refining Corp.

www.geibrefining.com

Booth 1122

Reclaim of Precious Metals; Precious Metal Products

Goodfellow Corporation

www.goodfellowusa.com

Metals and Materials for Research \& Development

Guangzhou Mikrouna Mechatronics

Booth 1029

Technology Co., Ltd.

www.mikrouna com

Glove Box; Gas Purification System

Hamamatsu Corporation

www.sales.hamamatsu.com

Quantum Yield Measurement; NIR; VUV; Electron

Detection and Imaging

HeatWave Labs Inc

www.cathode.com

Substrate Heaters; Cathodes; Electron Guns

Heidelberg Instruments Inc.

www. himt.de

Maskless Lithography Laser Writers; MicroPG101;

MicroPG501; DWL66FS; DWL2000; DWL4000

DWL8000; VPG1600

Herzan LLC

Booth 1304

www.herzan.com

Vibration Isolation; Acoustic Isolation; Environmental

Solutions; Site Survey Analysis

Hiden Isochema Ltd.

Booth 221

www.hidenisochema.com

Gas/Vapor Sorption Analyzers; Moisture Sorption

Analyzers

Hielscher USA, Inc

www.hielscher.com

Ultrasonic Mixers: Dispersion Technology;

Sonochemistry; Nano-Particle Milling; Sonocatalysis

TEM Specimen Holders
Hitachi High Technologies

America, Inc.

www.hitachi-hta.com

Electron Microscopes; Focused Ion Beam;

Sample Prep

HORIBA Scientific

Booth 901

Www.horiba.com/scientific

Raman; Spectroscopy; Ellipsometry

Hummingbird Scientific

www.hummingbirdscientific.com

Booth 524
Booth 207 
Huntington Mechanical

Booth 1001

Laboratories, Inc.

www.huntvac.com

Vacuum Manipulation \& Positioning; Vacuum

Chambers; Vacuum Valves \& Components

Hysitron, Inc.

www.hysitron.com

Booth 417

TI 950 Tribolndenter; TI 750 Ubi; TS 75 TriboScope

Hysitron, Inc.

Booth 324

www.hysitron.com

PI 95 TEM Picolndenter; PI 85 SEM Picolndenter; PI 87 SEM Picolndenter

\section{Image Metrology A/S}

Booth 1112

www.imagemet.com

SPIP Image Analysis Software

\section{Imina Technologies}

www.imina.ch

Micro- and Nanomanipulator; Electrical Prober;

Microscope Accessories

Inel, Inc.

Booth 325

www.inel us

X-ray Diffractometers ; Position Sensitive

Detectors; X-ray Generators

\section{INFICON}

Booth 613

www.inficon.com

Thin Film Deposition; Quartz Crystal Microbalance

Research; Vacuum Gauges and Components

Innovative Technology, Inc.

Booth 725

www.gloveboxes.com

Glove Boxes; Vapor Deposition Equipment;

Spin Coaters; Gas Purification Systems

InstruTech, Inc.

Booth 1019

www.instrutechinc.com

Vacuum Gauges; Vacuum Measurement Controllers;

Convection \& Ionization Vacuum Gauges

International Centre for Diffraction Booth 505

Data (ICDD)

www.icdd.com

X-Ray Powder Diffraction; Database; Software

Intlvac Thin Film

Booth 1313

www.intlvac.com

Ion Beam Etch Systems; Physical Vapor Deposition

ION-TOF USA, Inc.

Booth 818

www.iontofusa com

TOF-SIMS; AFM; Ion Scattering

IOP Publishing

Booth 110

publishing.iop.org

Leading journals including Nanotechnology

and Science \& Technology of Advanced Materials

iXRF Systems, Inc.

Booth 523

www.ixrfsystems.com

Microanalysis Systems (EDS);

XRF for the SEM; EBSD

Janis Research Company, LLC

Booth 930

www.janis.com

Micromanipulated Probe Stations; Cryostats;

Cryocoolers

Japan Advanced Institute

of Science and Technology

www.jaist.ac.jp/ms/English

Education; Research; Advanced Materials Science

Technology

Japan Science and Technology Agency Booth 204

www.jst.go.jp/tt/EN/univ-ip/cips/licensing.html

Technology Licensing for Categories including New

Materials, Electronic Devices \& Water Cleaning
Japan Society of Applied Physics

Booth 200

www.jsap.or.jp/english

Journals, including Applied Physics Express,

Japanese Journal of Applied Physics

JASCO

Booth 1124

Www.jascoinc.com

Raman; Near Field; FT-IR; UV-Vis/NIR; Thin-

Film Thickness Analysis; Materials Analysis;

Nanotechnology; Fluorescence

JEOL USA, Inc.

Booth 300

Www.jeolusa.com

TEM; SEM; Auger/MicroProbe

Kaufman \& Robinson, Inc.

Booth 222

www.ionsources.com

Ion Sources; Plasma Sources; Ion Beam Assist

Keithley Instruments, Inc.

Booth 601

www.keithley.com

Sensitive Electrical Measurement Instruments; I-V

Characterization Systems; DMMs and Power Supplies

Kimball Physics, Inc.

Booth 700

www kimballphysics com

UHV Electron and Ion Guns; Cathodes; Vacuum Chambers and Fittings

KLA-Tencor Corporation

Booth 600

www.kla-tencor.com

Stylus Profiler; Universal Microscope

KP Technology Ltd.

www.kelvinprobe.com

Scanning and UHV Work Function (Kelvin) Probes

Air Photoemission System

Kurt J. Lesker Company

Booth 400

www.lesker.com

Pure Targets and Materials; Vacuum Components;

Thin Film Deposition Systems: Target Bonding

Services, UHV Manipulation

Labtec Sales Partners LLC

Booth 721

www.labtecsp.com

Plasma Etch; Thin Film Deposition

and ALD; Maskless Lithography Systems

Lake Shore Cryotronics, Inc.

Booth 800

www lakeshore com

Probe Stations; Hall Effect Measurement Systems;

Cryogenic Instruments and Sensors

Leica Microsystems Inc.

Booth 316

www.leica-microsystems.com

Microscopes; Sample Preparation; Imaging Systems

LovaLite SAS

Booth 1312

www. Iovalite.com

Micro Optical Components; Nanotechnology;

Modeling Software

M. Braun, Inc.

Booth 317

www.mbraunusa.com

Gloveboxes \& Gas Purifiers; Vacuum Systems;

Solvent Purification Systems

MANTIS Deposition Ltd.

Booth 605

www.mantisdeposition.com

Nanoparticle Deposition; PVD; Thin Film

Deposition Tools

McCrone Group

www.mccrone.com

Microscopes; Analytical/Testing Services;

Benchtop SEM

MDC Vacuum Products, LLC

www.mdcvacuum.com

High \& Ultra High Vacuum Products;

Ceramic-to-Metal Feedthroughs; UHP Weldments
Metrohm USA, Inc.

Booth 322

www.metrohmusa.com

Electrochemical Systems; Impedance

Characterizations; Sensors

Mettler-Toledo, LLC

Booth 1011

www.mt.com

MP; DSC1; TGA/DSC1; Flash DSC

Michelson Prize \& Grants

michelson.foundanimals.org

Booth 730

Micro Materials Limited

Booth 1309

www micromaterials co.uk

NanoTest System; MicroTest System

Micro Photonics Inc.

Booth 1016

www.microphotonics.com

Micro CT; X-ray Cameras; X-ray Diffraction

MicroFab Technologies, Inc.

www.microfab.com

Precision Printing \& Microdispensing Systems

Jetting Components; Application Development

Services

Microtrac Inc.

Booth 906

www.microtrac.com

Particle Size; Imaging; Surface Area

MicroXact Inc.

www.microxact.com

Dicing and Polishing Services; MPS-200-C

and SPS-2600-PLUS Probe Systems

Milestone Inc.

Booth 830

Www.milestonesci.com

UltraWAVE; SynthWAVE; START

MMR Technologies, Inc.

www.mmr-tech.com

Hall Effect Measurement Systems; Seebeck Effect

Measurement Systems; Variable Temperature

Microprobe Systems

Molmex Scientific Inc.

www.molmexscientific.com

Small Angle X-ray Scattering; UItra-SAXS; USAXS;

X-ray Reflectivity; In Situ X-ray; Wide Angle X-ray

Scattering

Montana Instruments Corporation

Booth 821

www.montanainstruments.com

Cryostation

MTI Corporation

www.mtixtl.com

Multiple Zone Furnaces; High Vacuum Furnaces

PECVD Furnaces; Battery Research Equipment

MV Laboratories Inc.

www.mvlaboratories.com

High Purity Inorganics; Rare Earth Products; Precious Metal Products

NanoAndMore USA Inc.

www.nanoandmore com

AFM Probes; Digital Optical Microscopes; Digita

Holographic Microscopes

Nanofactory Instruments AB

Booth 904

www.nanofactory.com

In situ TEM Electrical, Mechanical

and Optical Probing Systems

Nanolnk, Inc.

Booth 811

www.nanoink.net

Micro/Nano Patterning; BioMEMS; Biomimetic

Surfaces for Cell Engineering

NANOLANE

Booth 710

www.nano-lane.com

Nanotechnology; Optical Microscopy;

Characterization Tools 
NanoMagnetics Instruments Ltd.

Booth 1101

www.nanomagnetics-inst.com

Atomic Force Microscope; Magnetic Force

Microscope; Nanopositioner

Nanomechanics, Inc.

Booth 1030

www.nanomechanicsinc.com

Analytical Services; In Situ Mechanical Testing;

Nanoindentation

\begin{tabular}{ll} 
Nanonics Imaging Ltd. & Booth 625 \\
www.nanonics.co.il & \\
NSOM/SNOM; AFM; AFM/Raman & \\
\hline
\end{tabular}

Nanoscience Instruments, Inc.

Booth 1113

www.nanoscience.com

Scanning Electron Microscopes; Atomic Force

Microscopes; 3D Optical Microscope

Nanosurf, Inc.

Booth 911

www.nanosurf.com

NaioAFM/STM; LensAFM; FluidFM

\section{Nanovea}

Booth 829

www.nanovea.com

ano/Micro/Macro Mechanical Tester;

3D Non-Contact Profilometers; Tribometers

\section{National Nanotechnology}

Infrastructure Network

Booth 217

www.nnin.org

Nanofabrication; Nanotechnology

\section{National Reconnaissance Office}

dii.westfields.net

Research \& Development Funding; Remote Sensing

Innovative Technology

\section{Nature Publishing Group}

Booth 124

www.nature.com

Medical \& Scientific Journals

\section{Neaspec GmbH}

www.neaspec.com

Aperturless NSOM/SNOM; Nanoscale Infrared

Spectroscopy; Raman Spectroscopy

Neocera, LLC

Booth 716

www.neocera.com

Pulsed Laser Deposition Systems; Pulsed Electron

Deposition Systems

Netzsch Instruments N.A. LLC

Booth 924

www.netzsch-thermal-analysis.com

Thermal Analysis; Thermal Conductivity; Thermal

Expansion; Calorimetry

NIST/CNST

Booth 220

www.cnst.nist.gov

Nanoscale Research Program; Nanofabrication

Facility; User Facility

\section{NIST/MSD}

Booth 216

www.nist.gov/srm

Standard Reference Materials; Data and Calibration Services

\section{NOF America Corporation}

Booth 1121

www.nofamerica.com

Phosphorylcholine (PC) Polymers; PC Derivatives

Nor-Cal Products, Inc.

Booth 425

www n-c.com

Vacuum Components \& Chambers;

Flanges \& Fittings; Valves

Novarials Corporation

Booth 1209

www.novarials.com

Various Nanowires: $\mathrm{TiO}_{2}$ Nanowires, $\mathrm{Al}_{2} \mathrm{O}_{3}$ Nanowires,

$\mathrm{MgO}$ Nanowires, $\mathrm{MnO}_{2}$ Nanowires, $\mathrm{MoO}_{3}$ Nanowires,

$\mathrm{V}_{2} \mathrm{O}_{5}$ Nanowires
NT-MDT Co.

Booth 211

www.ntmdt.com

SPM/AFM/STM; Raman TERS; Spectroscopy

\section{Oerlikon Leybold Vacuum}

www.oerlikon.com/leyboldvacuum

Thin Film Deposition Systems; Vacuum Pumps;

Leak Detectors

\section{Olympus America}

Booth 1013

www.olympusamerica.com

Inverted Metallographs; Upright Microscopes;

Stereo Microscopes; Filter Inspection

Omicron Nanotechnology USA, LLC Booth 817 Www.omicron-instruments.com

UHV SPM; Surface Science Instrumentation; MBE

OptiGrate Corporation

Booth 1108

www.optigrate.com

Volume Bragg Gratings; Ultra-Narrow-Band Notch

Filters; BragGrate Bandpass Filters

$\begin{array}{ll}\text { OriginLab Corporation } & \text { Booth } 616\end{array}$

www.originlab.com

Origin Software

Oxford Instruments America Inc.

Booth 816

www.oxford-instruments.com

ALD; Cryogenic Systems; Ion Beam; Microanalysis

Systems; Nanomanipulators; Plasma

\section{Oxford University Press \\ Booth 126}

www.oup.com/us

Publications

PANalytical Inc.

Booth 1000

www.panalytical.com

X-ray Diffraction (XRD); Computed Tomography

(CT); Small Angle X-ray Scattering (SAXS); X-ray

Fluorescence (XRF)

Park Systems Inc.

Booth 416

www. parkafm.com

Atomic Force Microscopes

\section{Particle Sizing Systems}

Booth 1022

www.pssnicomp com

AccuSizer SPOS; Nicomp DLS; Teclis

\section{Pascal Technologies Inc.}

Booth 822

www.pascaltechnologies.com

Vacuum Systems; Vacuum Equipment;

Leak Detectors

\section{PerkinElmer}

Booth 1100

www.perkinelmer.com

Spectrum Two; DSC

\section{Pfeiffer Vacuum}

Booth 711

www pfeiffer-vacuum com

Vacuum Pumps; Vacuum Instrumentation

\section{Photon etc.}

Booth 1311

www.photonetc.com

Hyperspectral Imager; Raman Hyperspectral Imager;

Tunable Filters and Sources; SWIR Camera

Photon Technology International, Inc. Booth 1008 www.pti-nj.com

Steady State Photoluminescence; Time-Resolved

Photoluminescence; NIR Photoluminescence

Photonic Cleaning Technologies, LLC Booth 728 www.photoniccleaning.com

Manufacturer of First Contact Polymer Products

Physical Electronics

Booth 712

\section{www.phi.com}

Scanning Auger; XPS; TOF-SIMS; Materials Analysis

Surface Analysis
Plasma-Therm LLC

Booth 618

Pww plasmatherm com

Semiconductor Manufacturing Equipment

Plasmaterials, Inc.

Booth 421

www.plasmaterials com

Sputtering Targets; Backing Plates; Evaporation

Materials; Bondin

Princeton Instruments

Booth 223

www.princetoninstruments.com

Spectroscopy Systems; CCD Cameras; Spectrometers

Princeton Scientific Corp.

www.princetonscientific.com

Metal Crystals; Laser Crystals; Superconductor

Wafers

Protochips, Inc.

Booth 302

www.protochips.com

Microscopes, Electron Microscopy and

Instrumentation; Nanotechnology; Biological,

Biomedical, Bio-related Sciences

PVD Products, Inc.

Booth 1200

www.pvdproducts.com

Pulsed Laser Deposition Systems; Sputtering

Systems; Evaporation Systems

Quantum Design, Inc.

Booth 511

www.qdusa.com

Physical Property and Magnetic Property

Measurement Systems

Quartz Imaging Corporation

www.quartzimaging.com

Digital Imaging Solutions; Laboratory Information

Management System; X-ray Microanalysis System

R.D. Mathis Company

Booth 718

www.rdmathis.com

Evaporation Sources; Evaporation Materials;

Power Supplies

Radiant Technologies, Inc.

Booth 420

www.ferrodevices.com

Ferroelectric Tester; Multiferroic Testers

Refining Systems, Inc.

www refiningsystems.com

Sputtering Targets; Crucibles and Dishes; Evaporation Materials; Wires and Tubing

Renishaw Inc

Booth 624

www.renishaw.com

Raman Microscopes; Spectrometers

Research and PVD Materials

Corporation

Booth 834

www.pvdmaterials.com

PVD Materials; Sputtering Targets; Evaporation

Sources

RHK Technology, Inc.

www.rhk-tech.com

SPM Universal Controls; UHV STM; UHV AFM/STM

Rigaku Americas Corporation

Booth 517

www.rigaku.com

X-ray Diffraction Systems; Small Angle

$\mathrm{X}$-ray Scattering Systems

Rigaku Innovative Technologies, Inc. Booth 522 www.rigaku.com

Multilayer X-ray Optics; X-ray Monochromators;

Microfocus X-ray Generators; X-ray CCD Cameras;

Rotary Motion Feedthroughs

Rigaku Raman Technologies

www. rigakuraman.com

First Guard Handheld Raman Spectrometer 
Rocky Mountain Vacuum Tech, Inc.

www.rmvac.com

Vacuum Systems; Vacuum Components

RSC Publishing

www.rsc.org/publishing

Journals; Books

\section{S.E.O. (Surface Electro Optics)}

Booth 1105

www.s-eo.com

Contact Angle Analyzer; Surface Tension

\section{SAGE Publications}

www.sagepub.com

Books; Journals

Scientific Instruments, Inc.

www.scientificinstruments.com

9700 Controller; SCM10; Diodes

\section{Seki Diamond Systems}

www.sekicvdsolutions.com

Microwave Plasma CVD Systems; Hot Filament

CVD Systems; Plasma CVD Systems

\section{SemiconSoft, Inc.}

Booth 1303

www.semiconsoft.com

MProbe Thin-film Measurement System;

TFCompanion Software

\section{Semicore Equipment Inc.}

Booth 1212

www.semicore.com

Sputtering Systems; Evaporation Systems; Custom

PVD Systems \& Components

Sentys Inc.

Booth 1208

www.sentys.com

Effusion Cells and MBE; RHEED; Low-Temperature STM

Solartron Analytical (AMETEK)

Booth 917

www.solartronanalytical.com

ModuLab MTS; 1260 Impedance Analyzer; Cryostats and Furnace

SonoPlot, Inc.

www.sonoplot.com

Microplotter; Printed Electronics; Materials Priner

SouthWest NanoTechnologies Inc. Booth 1116 www.swentnano.com

Single-wall Carbon Nanotubes (CNT); Specialty

Multiwall CNT; CNT-based Printable Inks;

CNT-based Smart Fabrics

SPECS Surface Nano Analysis, Inc. Booth 303 www.specs.com

JT Scanning Tunneling Microscope; NAP PHOIBOS

Energy Analyzer; Curlew SPM

SPEX SamplePrep LLC
www.spexsampleprep.com

Mixer/Mills; Freezer/Mills; X-Press

\section{SPI Supplies}

Booth 1202

Division of Structure Probe, Inc.

www.2spi.com

Supplies for Electron Microscopy; Plasma Etching Systems; TEM Membrane Grid

\begin{tabular}{lc}
$\begin{array}{l}\text { Springer } \\
\text { Www.springer.com }\end{array}$ & Booth $\mathbf{1 0 0}$ \\
Books; Journals; E-Books & \\
\hline $\begin{array}{l}\text { STAIB Instruments, Inc. } \\
\text { WwW.staibinstruments.com }\end{array}$ & Booth 717 \\
\begin{tabular}{l} 
RHEED; Spectrometers; Surface Analysis \\
\hline Strem Chemicals, Inc.
\end{tabular} \\
$\begin{array}{l}\text { Www.strem.com } \\
\text { High Purity Chemicals for R\&D; Nanomaterials; } \\
\text { MOCVD, CVD and ALD Precursors \& Bubblers; } \\
\text { Quantum Dots; Graphene }\end{array}$
\end{tabular}

Structured Materials Industries, Inc. Booth 607 www.structuredmaterials.com

MOCVD Systems; ALD Systems; Thin Film Deposition Components

Super Conductor Materials, Inc. Booth 620 www.scm-inc.com

Sputtering Targets; Evaporation Materials; Crucibles

SVT Associates, Inc.

Booth 404

www.svta.com

Molecular Beam Epitaxy; Pulsed Laser Deposition;

Atomic Layer Deposition

$\begin{array}{ll}\text { TA Instruments } & \text { Booth } 1021 \\ \text { www.tainstruments.com } & \end{array}$

DSC; TGA; Flash Diffusivity; Dilatometry

$\begin{array}{ll}\text { Taylor \& Francis-CRC Press } & \text { Booth } 116 \\ \text { www.taylorandfrancis.com } & \\ \text { Books; Journals; Online Products } & \end{array}$

Books; Journals; Online Products

Taylor Hobson, a Division of Ametek Booth 919 www.taylor-hobson.us

Optical Profiler

\section{TCI America}

Booth 1117

www.tciamerica.com

Fine Organic Chemicals; Bulk Chemicals

Lab Reagent R\&D Chemicals

Ted Pella, Inc.

www.tedpella.com

Vacuum Coaters; Calibration; Microscopy Sample

Preparation \& Supplies/Accessories

\section{TESCAN USA Inc}

www.tescan-usa.com

Electron Microscopes; SEM/FIB Microscopes;

Time of Flight TOF SIMS

The Mellen Company, Inc.

www.mellencompany.com

Furnaces; Crystal Growth; Custom Designs

Thermionics Vacuum Products

Booth 406

www.thermionics.com

Sample Handling; Electron Beam Sources;

Gate Valves

Thermo Scientific

Booth 422

www.thermoscientific.com/materialscience

Raman Microscope; XPS Spectrometer; X-Ray

Microanalysis System

\section{Toho Technology}

www.tohotechnology.com

FLX2320 Series; FLX3300 Series; FP Series Stylus

Profilers

Toshima Manufacturing Co., Ltd. Booth 1119 www.material-sys.com

Sputtering Targets; MOCVD Precursor; Functional Ceramics

\section{TREK, INC}

www.trekinc.com

High-Voltage Amplifiers; Electrostatic Voltmeters;

Surface/Volume Resistance Measurement

Trion Technology, Inc.

www.triontech.com

Reactive Ion Etcher (RIE); Plasma Enhanced Chemical

Vapor Deposition System (PECVD); Stripping Systems

TSI Inc.

Booth 921

www.tsi.com

Research Optical Particle Sizer; Water-Based

Condensation Particle Counters; Counters \& Sizers

UES, Inc.

Booth 1104

www.ues.com

RoboMet.3D; Advanced Coatings for Die Casting
ULVAC Technologies, Inc.

Booth 713

www.ulvac.com

Nano Particle Deposition Systems; Rapid Thermal

Annealing Equipment; Thermoelectric Measuring Equipment

United Mineral \& Chemical

Booth 1310

Corporation

www.umccorp.com

MBE Source Materials; MBE Equipment; Dopants

USHIO America, Inc.

Booth 1110

www.ushio.com

Electron Beam; Equipment; Custom Building

Vacuum Atmospheres Company Booth 1012

www.vac-atm.com

Glove Boxes \& Gas Purification; Thin Film Deposition; Solvent Purification

VAT, Inc.

Booth 916

Www.vatvalve.com

Vacuum Valves; Transfer Valves; Pressure Control System

VG Scienta, Inc.

Booth 703

www.vgscienta.com

Surface Analysis and UHV Systems \& Components

Valves; Sample Manipulators

Vigor Gas Purification

Booth 828

Technologies Inc.

www.vigor-glovebox.com

Glove Box; Gas Purification System; Solvent

Purification System

Wafer World Inc.

www.waferworld.com

Silicon Wafers; Germanium Wafers; GaAs Wafers

\section{Wiley}

Booth 112

www.wiley.com

Books; Journals; Online Resources

WiTec Instruments Corp.

Booth 610

www witec-instruments.com

Confocal Raman Microscopy; Scanning Near-Field

Microscopy; Atomic Force Microscopy

J.A. Woollam Company, Inc.

Booth 1004

www.jawoollam.com

Spectroscopic Ellipsometer

World Scientific Publishing Co.

Booth 1301

www.worldscientific.com

Books/eBooks; Journals/eJournals

XEI Scientific, Inc.

www.evactron.com

Remote Plasma De-contaminators for SEM, TEM and FIB Chambers; Sample Precleaning

XOS

Booth 1127

www.xos.com

Polycapillary Optics; Doubly-curved Crystal

X-Beam-X-ray Source with Optics

Xradia

Booth 920

www.xradia.com

UltraXRM; Versa XRM; UltraSPX

Yeagle Technology Inc.

www.ytionline.com Components

Zeta Instruments

www.zeta-inst.com

3D Optical Profiler; 3D Microscope; Film Thickness

Measurements

Zygo Corporation

www.zygo.com

Noncontact Inspection; Profilers; Metrology 